Pacific Journal of Mathematics

USING PREDICTION PRINCIPLES TO CONSTRUCT ORDERED 


\title{
USING PREDICTION PRINCIPLES TO CONSTRUCT ORDERED CONTINUA
}

\author{
STEPHEN WATSON
}

\begin{abstract}
In this paper, we show that the elements of a $\diamond$-sequence can be ordered lexicographically to produce an ordered continuum. An application of this idea answers a question of V. Malyhin and others: Is there a compact Hausdorff space in which no two points have equal character? We show that the consistency strength of the existence of such a space lies between that of an inaccessible and a Mahlo cardinal. We show that compactness is essential in this result by constructing, in ZFC, a $\sigma$-compact Hausdorff space in which no two points have equal character.
\end{abstract}

Let us begin with some definitions:

Definition 1. $\left\{f_{\alpha}: \alpha \in E\right\}$ is a $\searrow_{\kappa}(E)$-sequence (where $E \subset \kappa-\{0\}$ and $f_{\alpha}: \alpha \rightarrow 2$ ) if, for each $f: \kappa \rightarrow 2$, there is $\alpha \in E$ such that $f_{\alpha} \subset f$.

This is not exactly the standard definition (we use the characteristic functions of subsets of $\kappa$, we trap only once and do not require that $E$ be stationary or even cofinal in $\kappa$ ) but it is equivalent in most cases. The lexicographic ordering is not well defined because their domains are not equal. We need to subtract some $f_{\alpha} s$ which are "not needed". Let us fix this idea.

DeFINITION 2. $\left\{f_{\alpha}: \alpha \in E\right\}$ is a minimal $\diamond_{\kappa}(E)$-sequence if, whenever $F \subset E$ and $\left\{f_{\alpha}: \alpha \in F\right\}$ is a $\diamond_{\kappa}(F)$-sequence, $F$ must equal $E$.

This seems like a strong condition but it is not. We can subtract the $f_{\alpha}$ 's which are not needed.

LEMMA 1. If $\left\{f_{\alpha}: \alpha \in E\right\}$ is a $\diamond_{\kappa}(E)$-sequence, then there is $F \subset E$ such that

$$
\left\{f_{\alpha}: \alpha \in F\right\} \text { is a minimal } \diamond_{\kappa}(F) \text {-sequence. }
$$

Proof. The idea is to inductively subtract any $f_{\alpha}$ compatible with $f_{\beta}$ when $\beta<\alpha$. That is, $\alpha \in F$ iff, for each $\beta \in \alpha \cap F, f_{\beta} \cup f_{\alpha}$ is not a function. $\left\{f_{\alpha}: \alpha \in F\right\}$ is a $\diamond_{\kappa}(F)$-sequence since, for each $f: \kappa \rightarrow 2$ there is a minimal $\alpha \in E$ such that $f \uparrow \alpha=f_{\alpha}$. By the construction of $F$ and the 
minimality of $\alpha, \alpha \in F$. $\left\{f_{\alpha}: \alpha \in F\right\}$ is minimal since, for each $\alpha \in F$, we can find $f: \kappa \rightarrow 2$ such that $f \supset f_{\alpha}$. By the construction of $F$, there does not exist $\beta \in F$ such that $\beta \neq \alpha$ and $f \supset f_{\beta}$.

Minimality implies that the lexicographic ordering of a minimal $\diamond_{\kappa}(E)$-sequence is well-defined. Meanwhile, the $\diamond$ prediction property implies compactness.

Some notation is useful:

Let $\alpha * \beta$ be inf $\left\{\gamma: f_{\alpha}(\gamma) \neq f_{\beta}(\gamma)\right\}$.

The lexicographic ordering $\triangleleft$ is defined by $f_{\alpha} \triangleleft f_{\beta}$ iff $f_{\alpha}(\alpha * \beta)=0$.

LEMMA 2. If $\diamond=\left\{f_{\alpha}: \alpha \in E\right\}$ is a minimal $\diamond_{\kappa}(E)$-sequence, then the ordered space $X(\diamond)$ induced by the lexicographic ordering is compact.

Proof. We show that $\triangleleft$ is Dedekind-complete. That is, we show that for each $W \subset E$ such that $\alpha \in W, \beta \in E-W$ implies $f_{\alpha} \triangleleft f_{\beta}$, there is $\eta \in E$ such that, $\alpha \in W$ impies $f_{\alpha} \unlhd f_{\eta}$ and such that $\alpha \in E-W$ implies $f_{\eta} \unlhd f_{\alpha}$. Define $f: \kappa \rightarrow 2$ recursively by $f(\gamma)=1$ iff there is $\alpha \in W$ such that $f_{\alpha} \uparrow \gamma=f \uparrow \gamma$ and $f_{\alpha}(\gamma)=1$. This $f$ is "between" $\left\{f_{\alpha}: \alpha \in W\right\}$ and $\left\{f_{\alpha}: \alpha \in E-W\right\}$. Since $\left\{f_{\alpha}: \alpha \in E\right\}$ is a $\diamond$-sequence, there is $\eta \in E$ such that $f \supset f_{\eta}$. Fix $\alpha \neq \eta$.

If $\alpha \in W$ and $f_{\alpha} \triangleright f_{\eta}$ then $f_{\alpha}(\alpha * \eta)=1$ and $f_{\eta}(\alpha * \eta)=0$. By the construction of $f \supset f_{\eta}, f(\alpha * \eta)=1$ and that is a contradiction.

If $\alpha \in E-W$ and $f_{\eta} \triangleright f_{\alpha}$ then $f_{\eta}(\alpha * \eta)=1$ and $f_{\alpha}(\alpha * \eta)=0$. By the construction of $f \supset f_{\eta}$, there is $\beta \in W$ such that $f_{\beta} \uparrow \alpha * \eta=f \uparrow \alpha * \eta$ and $f_{\beta}(\alpha * \eta)=1$. Therefore $f_{\beta} \uparrow \alpha * \eta=f_{\alpha} \uparrow \alpha * \eta$ and $f_{\alpha}(\alpha * \eta)<$ $f_{\beta}(\alpha * \eta)$ so $f_{\alpha} \triangleleft f_{\beta}$ which is a contradiction.

The space $X(\diamond)$ may not be connected but it is dense-in-itself when $E$ consists of limit ordinals and that is what we need to make it connected.

LEMMA 3. If $\diamond=\left\{f_{\alpha}: \alpha \in E\right\}$ is a minimal $\diamond_{\kappa}(E)$-sequence and $E$ is a set of limit ordinals, then $X(\diamond)$ is dense-in-itself. To prove Lemma 3, one must essentially prove that the character of each point $f_{\alpha}$ in $X(\diamond)$ $\left(\chi\left(f_{\alpha}, X(\diamond)\right)\right)($ see $[1])$ is determined by its index:

LEMMA 4. If $\diamond=\left\{f_{\alpha}: \alpha \in E\right\}$ is a minimal $\diamond_{\kappa}(E)$-sequence and $\alpha \in E$ is a limit ordinal, then $\chi\left(f_{\alpha}, X(\diamond)\right)=\operatorname{cf} \alpha$.

Proof of Lemmas 3 and 4. It suffices to construct either (1) a $\triangleleft$-increasing $\operatorname{cf}(\alpha)$-sequence which converges to $f_{\alpha}$ and a $W \in[E]^{\leq \operatorname{cf}(\alpha)}$ such that, for each $\beta \in E$, with $f_{\beta} \triangleright f_{\alpha}$, there is $\gamma \in W$ such that $f_{\beta} \unrhd f_{\gamma} \triangleright f_{\alpha}$, 
or (2) a $\triangleleft$-decreasing $\operatorname{cf}(\alpha)$-sequence which converges to $f_{\alpha}$ and a $W \in$ $[E]^{\leq \operatorname{cf}(\alpha)}$ such that, for each $\beta \in E$ with $f_{\beta} \triangleleft f_{\alpha}$, there is $\gamma \in W$ such that $f_{\beta} \triangleleft f_{\gamma} \triangleleft f_{\alpha}$. Let $B$ be a cofinal subset of $\alpha$ of cardinality $\operatorname{cf}(\alpha)$. For each $\beta \in B$, construct $g_{\beta}: \kappa \rightarrow 2$ by $g_{\beta} \uparrow \beta=f_{\alpha} \uparrow \beta, g_{\beta}(\beta) \neq f_{\alpha}(\beta), g_{\beta} \uparrow \kappa-$ $(\beta+1) \equiv 1-f_{\alpha}(\beta)$ and find $\gamma(\beta) \in E: f_{\gamma(\beta)} \subset g_{\beta}$.

Find $i \in 2:\left|f_{\alpha}^{-1}(i) \cap B\right|=\operatorname{cf}(\alpha)$. If $i=0$, we construct (2) If $i=1$, we construct (1) Fix $i=0$ for this proof.

$\left\{f_{\gamma(\beta)}: \beta \in B ; f_{\alpha}(\beta)=0\right\}$ is the $\alpha$-sequence.

Let $\beta>\beta^{\prime}$ be fixed where $f_{\alpha}(\beta)=f_{\alpha}\left(\beta^{\prime}\right)=0$.

By the construction $f_{\gamma\left(\beta^{\prime}\right)}\left(\beta^{\prime}\right)=\mathrm{g}_{\beta^{\prime}}\left(\beta^{\prime}\right) \neq f_{\alpha}\left(\beta^{\prime}\right)=0$ and $f_{\gamma(\beta)}\left(\beta^{\prime}\right)=$ $g_{\beta}\left(\beta^{\prime}\right)=f_{\alpha}\left(\beta^{\prime}\right)=0$ while $f_{\gamma\left(\beta^{\prime}\right)} \uparrow \beta^{\prime}=g_{\beta^{\prime}} \uparrow \beta^{\prime}=f_{\alpha} \uparrow \beta^{\prime}=g_{\beta} \uparrow \beta^{\prime}=f_{\gamma(\beta)}$ $\uparrow \beta^{\prime}$. This implies $f_{\gamma(\beta)} \triangleleft f_{\gamma\left(\beta^{\prime}\right)}$ and so the $\alpha$-sequence is $\triangleleft$-decreasing. We show that the $\alpha$-sequence converges to $f_{\alpha}$. Otherwise $\exists \delta \in E \forall \beta \in f_{\alpha}^{-1}(0)$ $\cap B . f_{\alpha} \triangleleft f_{\delta} \triangleleft f_{\gamma(\beta)} \cdot\left|f_{\alpha}^{-1}(0) \cap B\right|=\alpha \Rightarrow \exists \beta>(\alpha * \delta): f_{\alpha}(\beta)=0 . f_{\gamma(\beta)} \subset g_{\beta}$ and $g_{\beta} \uparrow \beta=f_{\alpha} \uparrow \beta$ and $f_{\gamma(\beta)}$ incompatible with $f_{\alpha}$ implies $\gamma(\beta)>\beta$.

$f_{\gamma(\beta)} \uparrow(\alpha * \delta)+1=g_{\beta} \uparrow(\alpha * \delta)+1=f_{\alpha} \uparrow(\alpha * \delta)+1$ implies $f_{\gamma(\beta)} \uparrow$ $(\alpha * \delta)=f_{\delta} \uparrow(\alpha * \delta) . f_{\gamma(\beta)}(\alpha * \delta)=f_{\alpha}(\alpha * \delta)<f_{\delta}(\alpha * \delta)$ implies $f_{\gamma(\beta)} \triangleleft f_{\delta}$.

Let $W=\left\{\gamma(\beta): f_{\alpha}(\beta)=1, \beta \in B\right\}$. Suppose $\beta \in E$ and $f_{\beta} \triangleleft f_{\alpha}$. We find $\delta \cap W$ such that $f_{\beta} \triangleleft f_{\delta} \triangleleft f_{\alpha} . f_{\beta}(\beta * \alpha)=0$ and $f_{\alpha}(\beta * \alpha)=1$. Let $\delta=\gamma(\beta * \alpha), \quad f_{\delta} \subset g_{(\beta * \alpha)}$ and $f_{\alpha} \uparrow \beta * \alpha=g_{(\beta * \alpha)} \uparrow \beta * \alpha=f_{\alpha} \uparrow \beta * \alpha$ while $f_{\delta}(\beta * \alpha)=g_{(\beta * \alpha)}(\beta * \alpha) \neq f_{\alpha}(\beta * \alpha)=1$ so that $f_{\delta} \triangleleft f_{\alpha} . f_{\beta}\lceil\beta * \alpha$ $=f_{\alpha} \uparrow \beta * \alpha=f_{\delta} \uparrow \beta * \alpha$ while $f_{\beta}(\beta * \alpha)=f_{\delta}(\beta * \alpha)=0$ and $f_{\delta} \uparrow \delta-$ $((\beta * \alpha)+1) \equiv 1$ implies $f_{\beta} \unlhd f_{\delta}$. Lemma 3 enables us to make $X(\diamond)$ connected.

LEMMA 5. If $(X,<)$ is a compact ordered space which is dense-in-itself then letting an equivalence relation $\sim$ on $X$ be defined by $x \sim y$ if there is no $z \in X$ such that $x<z<y,(X / \sim,<)$ is a continuum.

The basic theorem can be proved now.

THEOREM 1. If there is a cardinal $\kappa$ and a set of regular infinite cardinals $E \subset \kappa$ such that $\diamond_{\kappa}(E)$ holds, then there is a ordered continuum with no two points of equal character.

Proof. If $\diamond$ is a $\diamond_{\kappa}(E)$-sequence, then Lemma 1 implies that we may assume $\diamond$ is minimal. Lemmas 2 and 3 implies that $X(\diamond)$ is compact and dense-in-itself. Lemma 4 implies that the character of a point is the regular infinite cardinal by which it is indexed. Lemma 5 produces an ordered continuum $X(\diamond) / \sim$ in which the character of an equivalence 
class is the maximum of the characters of its elements (since equivalence classes are finite), and the theorem is proved.

A partial converse can be proved.

THEOREM 2. If there is a compact Hausdorff space $X$ with no two points of equal character, then this is a cardinal $\kappa$ and a set of infinite cardinals $E \subset \kappa$ such that there is $a \diamond_{\kappa}(E)$-sequence.

Proof of Theorem 2. Let $\kappa=|X|$. Let $\left\{P_{\alpha}: \alpha \in \kappa\right\}$ enumerate $[X]^{2}$. Define $F \subset^{\kappa} 2$ and $\left\{U_{f}: f \in F\right\}$ a family of open sets such that

1. $\left|\bigcup\left\{U_{f \mid \beta} \beta<\operatorname{dom} f\right\}\right|>1$ iff $f \in F$

2. $f \in F \Rightarrow \bigcup\left\{U_{f \nmid \beta}: \beta<\operatorname{dom} f\right\}=\varnothing$

3. $f, f^{\prime} \in F,\left|f^{\prime}-f\right|=1$ and $f^{\prime} \supset f$ implies $\bar{U}_{f^{\prime}} \subset U_{f}$

4. $f, f^{\prime}, f^{\prime \prime} \in F,\left|f^{\prime}-f\right|=1=\left|f^{\prime \prime}-f\right|$ and $f^{\prime} \neq f^{\prime \prime}$ and $f^{\prime} \supset f, f^{\prime \prime}$ $\supset g$ implies $U_{f} \cap U_{f^{\prime}}=\varnothing$

5. $f \in F \Rightarrow\left|U_{f} \cap P_{\operatorname{dom} f}\right| \leq 1$

6. $U_{\varnothing}$ is an open set containing no isolated point and no point of character $\kappa$.

For any $g: \kappa \rightarrow 2$, there is a unique $x_{g} \in \bigcap\left\{U_{f}: f \in F, f \subset g\right\}$. If the character of $x_{g}$ is $\alpha_{g}<\kappa$ then let $f_{\alpha_{g}}: \alpha_{g} \rightarrow \alpha$ be defined by $f_{\alpha_{g}} \subset g$. This is well-defined since no two points have equal character. Let $E=\left\{\alpha_{g}\right.$ : $\left.g \in{ }^{\kappa} 2\right\}$.

This proof is a simple modification of P.291 of [1].

Corollary to Theorem 1. $(V=L+\exists$ Mahlo cardinal. $)$ There is an ordered continuum with no two points of equal character.

Proof. The definition of a Mahlo cardinal is a regular cardinal $\kappa$ which has a stationary subset $E$ of regular infinite cardinals. Under $V=L$, whenever $\kappa$ is a regular cardinal and $E$ is a stationary subset of $\kappa$ then $\diamond_{\kappa}(E)$ holds (see p. 181 of [2]).

Corollary to Theorem 2. ( $\exists$ weakly inaccessible cardinal.) Any compact Hausdorff space has two points of equal character.

Proof. Let $\kappa$ be the least cardinal such that there is a set of infinite cardinals $E \subset \kappa$ and a $\diamond_{\kappa}(E)$-sequence $\left\{f_{\alpha}: \alpha \in E\right\}$. If $\kappa$ is a successor, then $\kappa$ is not minimal. If $\kappa$ is a singular limit, then $\kappa$ is also not minimal but an argument is needed. 
Let $C$ be a closed unbounded set of order-type $\operatorname{cf}(\kappa)$.

Let $D$ be the set of limit points of $C$.

Any cardinal in $D$ has cofinality less than $\operatorname{cf}(\kappa)$; thus $D \cap E \subset \operatorname{cf}(\kappa)$.

Let $F=D-\operatorname{cf}(\kappa)$; thus $E \cap F=\varnothing$. If $\alpha \in F$, whenever possible, let $\alpha^{-}$be the greatest element of $F \cup\{0\}$ which is smaller than $\alpha$. For each $\alpha \in F$, by minimality, there is $g_{\alpha}:\left(\left\{\gamma \in \kappa: \alpha^{-} \leq \gamma<\alpha\right\}\right) \rightarrow 2$ which does not contain any $f_{\gamma} \uparrow \gamma-\alpha$ whenever $\alpha^{-} \leq \gamma<\alpha$.

Let $h: \kappa \rightarrow 2$ contain each $g_{\alpha} . h$ does not contain any $f_{\gamma}$ whenever $\gamma \in E$.

We demonstrate that compactness is an essential condition in these results by proving

THEOREM 3. (ZFC) There is a o-compact Hausdorff space $X$ in which no two points have equal character.

We need a set-theoretic lemma.

LeMMA 6. There is an infinite cardinal $\kappa$ such that $\boldsymbol{\aleph}_{\kappa}=\kappa$.

Proof. Induction on $\alpha \leq \omega_{1}$. Let $\kappa_{0}=\omega$. Let $\kappa_{\alpha+1}=\aleph_{\kappa_{\alpha}}$. Let $\kappa_{\alpha}=$ $\sup \left\{\kappa_{\beta}: \beta<\alpha\right\}$ when $\alpha$ is a limit. Let $\kappa=\kappa_{\omega_{1}}$.

We need to construct a tree

LEMMA 7. There is an infinitely branching tree $(T,<)$ of height $\omega$ such that (letting $t^{*}=\left\{t^{\prime} \in T: t^{\prime}>t\right.$ and level $t^{\prime}=$ level $\left.t+1\right\}$; the immediate successors of $t) t, t^{\prime} \in T$ and $t \neq t^{\prime}$ implies $\left|t^{*}\right| \neq\left|t^{\prime *}\right|$.

Proof. Construct the tree on $\kappa$ of Lemma 6 by induction on level. If level $n$ has been constructed, let $T_{n}$ be the set of nodes at height less than $n$, let $S_{n}$ be the set of nodes at height $n$, let $A$ be the set of cardinals less than $\kappa$.

We carry an induction hypothesis that $\left|A-\left\{\left|t^{*}\right|: t \in T_{n}\right\}\right|=\kappa$ and find an injection $\pi: S_{n} \rightarrow A-\left\{\left|t^{*}\right|: t \in T_{n}\right\}$.

Define $t \in S_{n}$ to have $\pi(t)$-many immediate successors.

Proof of Theorem 3. Topologize the tree of Lemma 7 by letting a neighborhood of $t \in T$ be defined in each $F \in\left[t^{*}\right]^{<\omega}$ by $U_{f}(t)=\{s \in T$ : (1) $s>t$ and (2) $u \in F$ implies $s \ngtr u\}$. $T_{n}$ is a compact subset of $T$ and so $T$ is $\sigma$-compact. 
The corollary to Theorem 2 was independently obtained by Peg Daniels. The author thanks the referee for many useful comments on the proof of Theorem 2 and the corollary to Theorem 2.

\section{REFERENCES}

[1] R. Engelking, General Topology, PWN, Polish Scientific Publishers, Warsaw, 1979.

[2] K. Kunen, Set Theory, North Holland, Amsterdam, 1983.

Received September 21, 1984. This work has been supported by the Natural Sciences and Engineering Research Council of Canada.

YORK UNIVERSITY

Downsview, OnTario, Canada M3J 1P3 


\section{PACIFIC JOURNAL OF MATHEMATICS EDITORS}

\author{
V. S. VARADARAJAN \\ (Managing Editor) \\ University of California \\ Los Angeles, CA 90024 \\ HERBERT ClEMENS \\ University of Utah \\ Salt Lake City, UT 84112 \\ R. FINN \\ Stanford University \\ Stanford, CA 94305
}

\author{
HERMANN FLASCHKA \\ University of Arizona \\ Tucson, AZ 85721 \\ RAMESH A. GANGOLLI \\ University of Washington \\ Seattle, WA 98195 \\ VAUghan F. R. JONES \\ University of California \\ Berkeley, CA 94720 \\ ROBION KIRBY \\ University of California \\ Berkeley, CA 94720
}

C. C. MOORE

University of California Berkeley, CA 94720

H. SAMELSON

Stanford University Stanford, CA 94305

HAROLD STARK

University of California, San Diego La Jolla, CA 92093

\section{ASSOCIATE EDITORS}
R. ARENS
E. F. BECKENBACH
B. H. NEUMANN
F. WOLF
K. YOSHIDA (1906-1982)

\section{SUPPORTING INSTITUTIONS}

UNIVERSITY OF ARIZONA

UNIVERSITY OF BRITISH COLUMBIA

CALIFORNIA INSTITUTE OF TECHNOLOGY

UNIVERSITY OF CALIFORNIA

MONTANA STATE UNIVERSITY

UNIVERSITY OF NEVADA, RENO

NEW MEXICO STATE UNIVERSITY

OREGON STATE UNIVERSITY
UNIVERSITY OF OREGON UNIVERSITY OF SOUTHERN CALIFORNIA

STANFORD UNIVERSITY

UNIVERSITY OF HAWAII

UNIVERSITY OF TOKYO

UNIVERSITY OF UTAH

WASHINGTON STATE UNIVERSITY

UNIVERSITY OF WASHINGTON 


\section{Pacific Journal of Mathematics}

Vol. 125, No. 1 September, 1986

Gilles Christol, Fonctions et éléments algébriques $\ldots \ldots \ldots \ldots \ldots \ldots \ldots \ldots$

Jo-Ann Deborah Cohen, Extensions of valuation and absolute valued

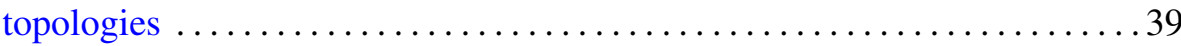

Miriam Cohen, Smash products, inner actions and quotient rings . . . . . . 45

Mikio Furushima, On the singular $K-3$ surfaces with hypersurface

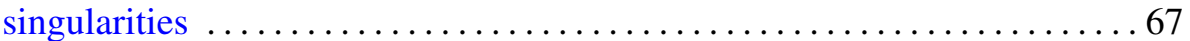

Gerhard Gierz and Boris Shekhtman, A duality principle for rational

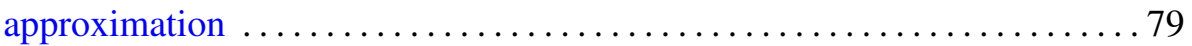

Anthony Wood Hager, A description of HSP-like classes, and

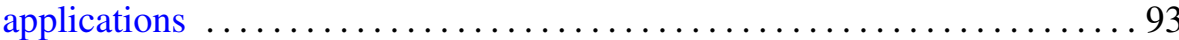

George Alan Jennings, Lines having high contact with a projective

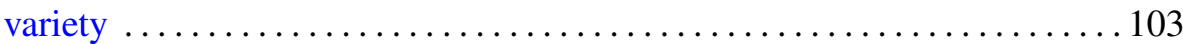

John Lott, Eigenvalue bounds for the Dirac operator . . . . . . . . . . . 117

Denis Laurent Luminet, A functional calculus for Banach PI-algebras . . . . 127

Shizuo Miyajima and Noboru Okazawa, Generators of positive

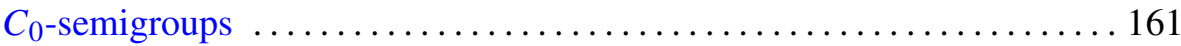

Takemi Mizokami, On functions and stratifiable $\mu$-spaces $\ldots \ldots \ldots \ldots \ldots 177$

Jeff Parker, 4-dimensional $G$-manifolds with 3-dimensional orbits . . . . . 187

Elias Saab and Paulette Saab, On Peł czyński's properties (V) and (V*) . . 205

Elmar Schrohe, The symbols of an algebra of pseudodifferential operators

Aart van Harten and Els Vader-Burger, Approximate Green functions as a tool to prove correctness of a formal approximation in a model of competing and diffusing species

Stephen Watson, Using prediction principles to construct ordered

continua 